\title{
The nominal/FM Yoneda Lemma
}

\author{
R. L. Crole*(iD) \\ School of Informatics, University of Leicester, University Road, Leicester, LE1 7RH, UK \\ *Corresponding author. Email: rlc3@le.ac.uk
}

(Received 18 June 2020; revised 29 December 2020; accepted 30 December 2020)

\begin{abstract}
This paper explores versions of the Yoneda Lemma in settings founded upon FM sets. In particular, we explore the lemma for three base categories: the category of nominal sets and equivariant functions; the category of nominal sets and all finitely supported functions, introduced in this paper; and the category of FM sets and finitely supported functions. We make this exploration in ordinary, enriched and internal settings. We also show that the finite support of Yoneda natural transformations is a theorem for free.
\end{abstract}

Keywords: Yoneda Lemma; nominal sets; FM sets

\section{Introduction}

The purpose of this paper is to establish some instances of the Yoneda Lemma in settings involving both nominal sets and FM sets. We, therefore, assume readers are fluent in the basics of category theory (Lane 1998) and in particular the Yoneda Lemma. We also assume that readers have some knowledge of basic enriched and internal category theory, although we have included an Appendix devoted to the notation we shall use. In relation to the enriched setting, we will work with both the weak and strong enriched Yoneda Lemmas as presented by Kelly in (1982). We also assume readers are familar with nominal and FM sets; for an excellent and comprehensive introduction to nominal and FM sets, see the monograph by Pitts (2013) and Gabbay's thesis (http://www.gabbay.org.uk/papers.html\#thesis). While the Yoneda Lemma is the central focus of the paper, we would like to begin with background material that provides a basis and some motivation for our results.

This paper originates from thinking about Nominal Equational Logic introduced in Clouston and Pitts (2007) and also Gabbay and Mathijssen (2009). (Note that we are not referring to the system Clouston 2011). Let us recall some of the basic ingredients of Nominal Equational Logic (NEL), with the notation below based on Figure 5 of Clouston and Pitts (2007). NEL is a simple type theory that provides equational reasoning (over algebraic expressions). There are three forms of judgement, although in the referenced Figure 5, one judgement form is written down in terms of another, so it appears as though there are just two judgements. One form is that of freshness judgements $a$ \# $M$ in side conditions which assert that the atom $a$ is fresh for the expression $M$ (see, for example, a vital side condition of the (ATM-INTRO) rule): NEL expressions $M$ are elements of FM sets, and the (syntactic) freshness judgement above is made in this context. In addition, there is a formal relational judgement $a \overrightarrow{\#} M=M^{\prime}$, which encodes $\mathrm{M}$ semantically equals $\mathrm{M}$, and indirectly (the third form) the atom $a$ is semantically fresh for $\mathrm{M}$ via the equation for reflexivity. One can then give denotations to expressions in the category of FM sets, which we abbreviate to $\mathscr{F} \mathscr{M} \mathscr{S e t}$, with the deduction system of NEL being sound and complete.

(C) The Author(s), 2021. Published by Cambridge University Press. This is an Open Access article, distributed under the terms of the Creative Commons Attribution licence (http://creativecommons.org/licenses/by/4.0/), which permits unrestricted re-use, distribution, and reproduction in any medium, provided the original work is properly cited. 
The author along with Frank Nebel introduced the Nominal Lambda Calculus NLC in Crole and Nebel (2013) (again, no deep knowledge is required for reading this paper). NLC is a (dependent) type theory that extends Nominal Equational Logic with higher order functions, much as the lambda calculus LC extends 'standard' equational EL. Now in Crole (1996), Crole proves (amongst other things) that LC is a conservative extension of EL by using logical relations categorical gluing; for functional gluing see, for example, Lambek and Scott (1986) (or Carboni and Johnstone 1995; Moerdijk 1982 for topos specific background).

Let us say a little more about the ideas at the heart of gluing proofs of such conservative extensions. Suppose that $T h$ is an EL theory and that $T h^{\prime}$ is the LC theory generated by taking the ground types and function symbols of $T h^{\prime}$ to be those of $T h$. Let $E(x: \gamma): \gamma^{\prime}$ be a $T h^{\prime}$ expression where $\gamma, \gamma^{\prime}$ are ground types. The statement that $T h^{\prime}$ is a conservative extension of $T h$ means that there is a Th expression $M(x: \gamma): \gamma^{\prime}$ for which $E=M$ is provable in $T h^{\prime}$; roughly speaking such $E$ can always be $\beta \eta$-reduced to $M$.

Let us write $\mathscr{C}(T h)$ for the classifying category of $T h$ (see, for example, Crole 1993 or Johnstone 2002). One may prove that $T h^{\prime}$ is a conservative extension of $T h$ categorically by establishing that there is a full embedding $I: \mathscr{C}(T h) \rightarrow \mathscr{C}\left(T h^{\prime}\right)$. If so, then $\mathscr{C}(T h)\left(\gamma, \gamma^{\prime}\right) \cong_{1}$ $\mathscr{C}\left(T h^{\prime}\right)\left(I \gamma, I \gamma^{\prime}\right)$. Thus, a morphism $E: I \gamma \rightarrow I \gamma^{\prime}$ in the classifying category $\mathscr{C}\left(T h^{\prime}\right)$ is formally equal to $I M$ where $M: \gamma \rightarrow \gamma^{\prime}$ (and $E=I M$ means $E=M$ in $T h^{\prime}$ ).

Suppose that $\mathscr{D}$ is a category, with objects $A$ and $B$, and that $\mathscr{V}$ a cartesian closed category such as $\mathscr{S}$ et or $\omega \mathscr{C} \mathscr{P} \mathscr{O}$. The Yoneda Embedding is a functor $Y: \mathscr{D}^{\circ p} \rightarrow \mathscr{V}^{\mathscr{D}}$ where $Y: A \mapsto\left(Y^{A}: \mathscr{D} \rightarrow\right.$ $\mathscr{V})$. Crole has shown (Crole 1993, 1996) that the existence of $\cong_{1}$ can be reduced to showing that the functor category $\mathscr{V}^{\mathscr{D}}$ is also a ccc and that $\mathscr{D}(A, B) \cong_{2} \mathscr{V}^{\mathscr{D}}\left(Y^{A}, Y^{B}\right)$. Now, isomorphisms such as $\cong_{2}$, and the cartesian closure of functor categories like $\mathscr{V}^{\mathscr{D}}$, can be established using instances of the (enriched) Yoneda Lemma.

Thus, to develop an FM version of gluing, we would like to have versions of the Yoneda Lemma and cartesian closure when $\mathscr{V}$ is

- Nom - the category of nominal sets and equivariant functions (Pitts 2003, 2013);

- $\mathscr{F} \mathscr{M} \mathscr{N o m}$ - the category of nominal sets and all finitely supported functions, introduced in this paper; and

- $\mathscr{F} \mathscr{M} \mathscr{S}$ et - the category of FM sets (Clouston 2011, 2014) and finitely supported functions,

where there are inclusions $\mathscr{N o m} \hookrightarrow \mathscr{F} \mathscr{M} \mathscr{N o m} \hookrightarrow \mathscr{F} \mathscr{M} \mathscr{S e t}$. The first is a luff subcategory, the second a full subcategory.

This paper studies the Yoneda Lemma for these categories. In working with our future applications, we found that we needed to perform various concrete computations, which were only enabled by unravelling the abstract details of the enriched category theory. It seemed interesting to provide a short summary of the results.

In more detail: In Section 2, we summarise our basic notation; and we recall that both $\mathscr{F} \mathscr{M} \mathscr{N o m}$ and $\mathscr{F} \mathscr{M} \mathscr{S e t}$ are incomplete. Incompleteness is important since a strong Yoneda Lemma requires completeness. We also look at cartesian closure of the $\mathscr{V}$ categories. In Sections 3 and 4, we present versions of the weak and strong Yoneda Lemma. In Section 5, we study some results that follow from the weak and strong Yoneda Lemmas, and further, although there is no strong Yoneda Lemma for $\mathscr{F} \mathscr{M} \mathscr{N}$ om and $\mathscr{F} \mathscr{M} \mathscr{S e t}$, each has an internal version. In Section 6, we prove the cartesian closure of the categories which we require in nominal gluing, by applying our Yoneda results. In Section 7 we conclude. 


\section{Some Notation and a Routemap}

In this section, we summarise the notation we use, and review a few known results that help to provide a routemap for our results about the Yoneda Lemma.

\subsection{Notation}

We introduce some basic notation. Suppose that $f: X \rightarrow Y$ is a function between (ZF or FM) sets. We may write $f(x \in X) \in Y$ instead of $f(x)$ to indicate the source set for $x$ and target set for $f(x)$. We occasionally use the notation $f: x \mapsto f(x)$. Let $X^{\prime} \subseteq X$ be a subset. We shall write $f_{\left.\right|_{X^{\prime}}}$ for the function restriction $X^{\prime} \rightarrow Y$ whose graph is that of $f \cap\left(X^{\prime} \times Y\right)$. If, further, the image $\operatorname{im}(f)$ of $f$ is a subset of $Y^{\prime} \subseteq Y$, then we sometimes write $f_{\left.\right|_{X^{\prime}}}: X^{\prime} \rightarrow Y^{\prime}$ to indicate this. If $x \in X^{\prime}$ we often write just $f(x)$ instead of $f_{\left.\right|_{X^{\prime}}}(x)$.

Let $\mathbb{A} \stackrel{\text { def }}{=}\left\{a_{1}, \ldots, a_{k}, \ldots\right\}$ be an enumerated infinite set of atoms (names) (Gabbay and Pitts 2002). We write $\pi, \pi^{\prime}$ or similar for any permutation on $\mathbb{A}$ with finite domain; and $\tau, \tau^{\prime}$ for transpositions. Perm denotes the set of such permutations (equivalently those generated by transpositions $\tau_{a, b}=(a b)$ ). The composition of $\pi$ and $\pi^{\prime}$, with $\pi^{\prime}$ applied first, is denoted by $\pi \circ \pi^{\prime}$ or $\pi \pi^{\prime}$. If $X=(|X|, \cdot)$ is a nominal or FM set, we write $\pi \cdot x$ for the action of $\pi$ on $x$; we sometimes abuse notation and write $\pi \cdot(x \in X)$ for the action on $x$ if we wish to explicitly alert the reader to the set $X$ of which $x$ is a member. The space of finitely supported functions from (nominal or FM sets) $X$ to $Y$ is denoted by $X \Rightarrow_{f_{s}} Y$. If $X$ is an FM set, and $x \in X$, we write $\operatorname{supp}(x)$ for the support of $x$, and $a \# x\left(\tau_{a, b} \# x\right)$ to denote that $a \notin \operatorname{supp}(x)(a, b \notin \operatorname{supp}(x))$. We work with the standard definition of support: $S \subseteq \mathbb{A}$ supports $x \in X$ just in case

$$
(\forall \pi)((\forall a \in S)(\pi \cdot a=a) \Longrightarrow \pi \cdot x=x)
$$

or equivalently $(\forall a, b \in \mathbb{A})\left(a, b \notin S \Longrightarrow \tau_{a, b} \cdot x=x\right)$. Clearly for some $y \in Y$, supp $(y)$ supports $x \in$ $X$ just in case $(\forall a, b \in \mathbb{A})\left(\tau_{a, b} \# y \Longrightarrow \tau_{a, b} \cdot x=x\right)$. We write $X_{e s}$ for the set (in fact nominal subset) of emptily supported elements of $X$. Of course $\left|X_{e s}\right|=|X|_{e s} \stackrel{\text { def }}{=}\{x \in X \mid(\forall \pi)(\pi \cdot x=x)\}$. If $S$ is a set, we write $S<\infty$ as a notation for $S$ is finite. We will make use of the following trivial lemma (easy proof omitted).

Lemma 1. Suppose that $X=(|X|, \cdot)$ is a nominal set, and $\phi:|X| \cong \mathscr{S e t}_{\mathrm{et}} S: \psi$ is an isomorphism (bijection) of sets. Then $S$ is also a nominal set with the canonical permutation action $\pi * s \stackrel{\text { def }}{=} \phi(\pi$. $\psi(s))$, where we have $\operatorname{supp}(s)=\operatorname{supp}(\psi(s) \in X)$, and further $\Phi: X \cong_{\mathscr{N} o m}(S, *): \Psi$.

\section{$2.2 \mathscr{F} \mathscr{M} \mathscr{N o m}$ and $\mathscr{F} \mathscr{M}$ Set are not complete}

Recall that the strong Yoneda Lemma, for any $\mathscr{V}$-enriched functor $F: \mathscr{C} \rightarrow \mathscr{V}^{\text {er }}$ where $\mathscr{V}^{\text {er }}$ is $\mathscr{V}$ enriched over itself, stipulates that $\mathscr{V}$ is complete. However, it is well known that $\mathscr{F} \mathscr{M} \mathscr{S}$ et is not complete; see, for example, Gabbay (http://www.gabbay.org.uk/papers.html\#thesis). Arguably, the fundamental idea behind the proof goes all the way back to the original work of Mostowski (1939); the very same idea applies to $\mathscr{F} \mathscr{M} \mathscr{N}$ om. However, to make our paper self-contained, we outline the incompleteness of $\mathscr{F} \mathscr{M} \mathscr{N}$ om.

First, let us consider how products are defined in $\mathscr{N}$ om. Let $I$ be any (indexing) set. Then the product $\Pi_{i \in I} A_{i}$ consists of families $\left(a_{i} \in A_{i} \mid i \in I\right)$ with finite support, where the permutation action on the family is pointwise. Indeed, suppose we are given a family of morphisms $f_{i}: X \rightarrow$ $A_{i}$ for each $i \in I$. Since in $\mathscr{N}$ om each morphism $f_{i}$ is equivariant, we have $\operatorname{supp}\left(f_{i}(x)\right) \subseteq \operatorname{supp}(x)$, with $\operatorname{supp}(x)$ finite. Thus, the family $\left(f_{i}(x) \mid x \in X\right)$ is an element of the product object and so we may define a universal morphism $h: X \rightarrow \Pi_{i \in I} A_{i}$ by $h(x) \stackrel{\text { def }}{=}\left(f_{i}(x) \mid x \in X\right)$ with $p r_{i} \circ h=f_{i}$ for each $i \in I$. 
Crucially, the assertion $\operatorname{supp}\left(f_{i}(x)\right) \subseteq \operatorname{supp}(x)$ depends on the equivariance of the $f_{i}$. In $\mathscr{F} \mathscr{M} \mathscr{N o m}$, we know only that morphisms are finitely supported, and so it is not clear that (such) a universal morphism, for (such) a product object, would be well defined. Now, of course, there might be an alternative construction of product objects in $\mathscr{F} \mathscr{M} \mathscr{N o m}$. However, this is not the case.

Proposition 2. $\mathscr{F} \mathscr{M} \mathscr{N} o m$ is not complete: in fact it does not have products. A prooffor $\mathscr{F} \mathscr{M} \mathscr{S e t}$ is analogous. Nom is complete.

Proof. We assume, for a contradiction, that $\mathscr{F} \mathscr{M} \mathscr{N}$ om is complete. Consider $P \stackrel{\text { def }}{=} \Pi_{i \in \omega} \mathbb{A}$ (that is we take the product of $\omega$ copies of $A$ ) with projections $p r_{i}: P \rightarrow \mathbb{A}$. Note that, by definition, all projection maps $p r_{i}$ must be finitely supported. We now recursively select atoms from $A$ such that $a_{1} \notin \operatorname{supp}\left(p r_{1}\right)$ and for $i>1$ we pick $a_{i} \notin \bigcup_{j \leq i} \operatorname{supp}\left(p r_{j}\right) \cup\left\{a_{j} \mid j<i\right\}$. Hence, we have infinitely many distinct atoms $a_{i}$, which satisfy $\forall j \in \omega . \forall i \geq j . a_{i} \# p r_{j}$.

Take morphisms $\hat{a}_{i}: 1 \rightarrow$ A defined by $\hat{a}_{i}(*) \stackrel{\text { def }}{=} a_{i}$. Each function $\hat{a}_{i}$ is finitely supported by $\left\{a_{i}\right\}$. By the universal property for products, there exists a finitely supported morphism $h: 1 \rightarrow P$ in $\mathscr{F} \mathscr{M} \mathscr{N} o m$ where $\hat{a}_{i}=p r_{i} \circ h$. Given that the support of $h$ is finite, there exists $r \in \omega$ such that for all $s \geq r$ we have $a_{s} \# h$. Choose one such $s_{0}$. Then the atoms $a_{s_{0}}$ and $a_{s_{0}+1}$ are both fresh for $p r_{s_{0}}$. If $\tau \stackrel{\text { def }}{=}\left(a_{s_{0}} a_{s_{0}+1}\right)$ then $\tau \cdot h=h$ and $\tau \cdot p r_{s_{0}}=p r_{s_{0}}$. Hence, the contradiction

$$
\begin{aligned}
a_{s_{0}}=\hat{a}_{s_{0}}(*)=\left(p r_{s_{0}} \circ h\right)(*) & =\left(\tau \cdot p r_{s_{0}} \circ \tau \cdot h\right)(*) \\
& =\left(\tau \cdot\left(p r_{s_{0}} \circ h\right)\right)(*) \\
& =\left(\tau \cdot \hat{a}_{s_{0}}\right)(*)=\tau \cdot\left(\hat{a}_{s_{0}}(*)\right)=\tau \cdot a_{s_{0}}=a_{s_{0}+1} .
\end{aligned}
$$

Since $\mathscr{F} \mathscr{M} \mathscr{N}$ om is a full subcategory of $\mathscr{F} \mathscr{M} \mathscr{S e t}$ the result is immediate: $\mathbb{A}$ is indeed an FM set, and the constructions (of morphism cones, cocones and universals) above remain unchanged in $\mathscr{F} \mathscr{M} \mathscr{S e t}$ by fullness. It is well known that $\mathscr{N} o m$ is complete.

Remark 3. In passing, we remark that $\mathscr{F} \mathscr{M} \mathscr{S}$ et and $\mathscr{F} \mathscr{M} \mathscr{N}$ om have all limits in the case that the limiting object has finite support; see Gabbay (http://www.gabbay.org.uk/papers.html\#thesis).

Proof. We consider $\mathscr{F} \mathscr{M}$ Set only. Define

$$
L \stackrel{\text { def }}{=}\left\{\left(x_{I} \mid I\right) \mid x_{I} \in D I \wedge\left(\forall u: I \rightarrow I^{\prime}\right)\left((D u)\left(x_{I}\right)=x_{I}^{\prime}\right) \wedge \bigcup_{I} \operatorname{supp}\left(x_{I}\right)<\infty\right\} .
$$

Then $L$ is an FM set. The action $\pi \cdot L$ is defined pointwise on tuples (more correctly, it is inherited via the FM hierarchy); and each tuple is finitely supported by construction, with $\bigcup_{I}$ $\operatorname{supp}\left(\pi \cdot x_{I}\right)=\pi \cdot \bigcup_{I} \operatorname{supp}\left(x_{I}\right)$. The canonical projections $\operatorname{pr}_{I}: L \rightarrow D I$ are easily seen to be equivariant. Take any fs-cone $\left(f_{I}: C \rightarrow D I \mid I\right)$ and define $h: C \rightarrow L$ by $h(x \in C) \stackrel{\text { def }}{=}\left(f_{I}(x) \mid I\right) . h$ is finitely supported since $\bigcup_{I} \operatorname{supp}\left(f_{I}\right)<\infty$. And $h(x) \in L$ since it is supported by $\bigcup_{I} \operatorname{supp}\left(f_{I}(x)\right)=$ $\bigcup_{I} \operatorname{supp}\left(f_{I}\right) \cup \operatorname{supp}(x)<\infty$.

The next Proposition 4 provides a further simple guide to which forms of the Yoneda Lemma we can expect to hold.

Proposition 4. Each of the categories $\mathscr{N o m}, \mathscr{F} \mathscr{M} \mathscr{N o m}$ and $\mathscr{F} \mathscr{M} \mathscr{S e t}$ has finite products, and each is cartesian closed with the exponential of $X$ and $Y$, in each case, $X \Rightarrow_{f_{s}} Y$. 
Proof. It is well known that $\mathscr{N}$ om is a cartesian closed category. We briefly explore the proof that $\mathscr{F} \mathscr{M} \mathscr{N o m}$ is a ccc: the proof for $\mathscr{F} \mathscr{M} \mathscr{S e t}$ is analogous. Since $\mathscr{N o m}$ is a luff subcategory of $\mathscr{F} \mathscr{M} \mathscr{N} o m$, we may take the exponential of $X, Y \in o b \mathscr{F} \mathscr{M} \mathscr{N} o m$ to be the nominal set $X \Rightarrow_{f_{s}} Y$. Then the equivariant evaluation function $\mathrm{ev}:\left(X \Rightarrow_{f_{s}} Y\right) \times X \rightarrow Y$ of $\mathscr{N o m}$ is also the evaluation function in $\mathscr{F} \mathscr{M} \mathscr{N o m}$ : If $m: Z \times X \rightarrow Y$ is finitely supported, provided that $\lambda m: Z \rightarrow X \Rightarrow_{f_{s}} Y$ specified by $\lambda m(z)(x) \stackrel{\text { def }}{=} \lambda x . m(z, x)$ is well defined in $\mathscr{F} \mathscr{M} \mathscr{N} o m$, all remaining details trivially mimic those of $\mathscr{N} o m$. First, $\lambda m$ is finitely supported by $\operatorname{supp}(m)$ which we check in detail: $(\tau \cdot \lambda m)(z)(x)=(\tau \cdot(\lambda m(\tau \cdot z)))(x)=\tau \cdot((\lambda m(\tau \cdot z))(\tau \cdot x))=\tau \cdot(m(\tau \cdot z, \tau$. $x))=\tau \cdot(m(\tau \cdot(z, x))=(\tau \cdot m)(z, x)=m(z, x)=\lambda m(z)(x)$. Second, $(\lambda m)(z)$ is finitely supported in $X \Rightarrow_{f_{s}} Y$ by $\operatorname{supp}(m) \cup \operatorname{supp}(z)$ : we omit the details which are similar to the previous calculation.

Side remark: note carefully that in $\mathscr{N}$ om such internal homs do not correspond to the external hom $\operatorname{Nom}(X, Y)$ of equivariant functions from $X$ to $Y$.

From Proposition 4 and from general results about enriched category theory (Kelly 1982, 2005), we know that if $\mathscr{V}$ is any of $\mathscr{N} o m, \mathscr{F} \mathscr{M} \mathscr{N} o m$ and $\mathscr{F} \mathscr{M} \mathscr{S}$ et, $\mathscr{V}$ enriches over itself as $\mathscr{V}^{e r}$ with $\mathscr{V}^{e r}(X, Y) \stackrel{\text { def }}{=} X \Rightarrow_{f_{s}} Y$. It, therefore, follows from enriched category theory that there is a weak Yoneda Lemma for each $\mathscr{V}^{e r}$. And we already noted that Proposition 2 implies that $\mathscr{F} \mathscr{M} \mathscr{N} o m$ and $\mathscr{F} \mathscr{M} \mathscr{S e t}$ will not enjoy strong versions of the Yoneda Lemma, but since $\mathscr{N o m}$ is complete, we know there is a corresponding strong Yoneda Lemma.

In Sections 3 and 4, we study such weak and strong Yoneda Lemmas, and show that we can also prove some interesting subsidiary results. We will explore the Yoneda Lemma, both in the abstract, and also by looking at bare-hands proofs: We will see that a bare-hands approach throws light on the intricacies of the abstract machinery involved, and indeed yields interesting and useful results that extend those of the abstract category theory.

Please scan the Appendix notation on page 1022 if required since the remaining sections draw heavily on enriched and internal category theory. I have tried to use Kelly's notation as much as possible.

\section{The Weak Yoneda Lemma}

\subsection{Weak Yoneda}

Let $\mathbb{C}$ be a $\mathscr{N} o m$-enriched category and $F: \mathbb{C} \rightarrow \mathscr{N}_{o m}{ }^{e r}$ a $\mathscr{N} o m$-enriched functor. For $A \in o b \mathbb{C}$ there is an enriched functor $\mathbb{C}^{A}: \mathbb{C} \rightarrow \mathscr{N}_{o m}{ }^{e r}$. Recall that this is specified by $\mathbb{C}^{A}: B \in o b \mathbb{C} \mapsto$ $\mathbb{C}(A, B) \in o b \mathscr{N}_{o m}$, and the morphism

$$
\mathbb{C}_{B, B^{\prime}}^{A}: \mathbb{C}\left(B, B^{\prime}\right) \longrightarrow \mathscr{N o m}^{\text {er }}\left(\mathbb{C}(A, B), \mathbb{C}\left(A, B^{\prime}\right)\right) \stackrel{\text { def }}{=} \mathbb{C}(A, B) \Rightarrow_{f_{s}} \mathbb{C}\left(A, B^{\prime}\right)
$$

is defined using Proposition 4 , to be $\lambda M_{A, B, B^{\prime}}$, the mate of the composition morphism for $\mathbb{C}$ (see Section A.1 if required). If $b \in \mathbb{C}\left(B, B^{\prime}\right)$ then we will sometimes denote $\mathbb{C}_{B, B^{\prime}}^{A}(b) \stackrel{\text { def }}{=}(\lambda M)(b)$ by one of the following alternative notations $\mathbb{C}^{A}(b) \equiv \mathbb{C}(A, b) \equiv b_{*}$. From the proof of Proposition 4 , $\operatorname{supp}\left(b_{*}\right)=\operatorname{supp}(b)$ since $M$ is equivariant in $\mathscr{N} o m$.

We will need to consider various kinds of 'natural families' of morphisms, such as those arising as (the components of) natural transformations between functors. We introduce some more notation which will play a useful role in this section. We define, where the products are in Set and of underlying function spaces,

$$
\begin{aligned}
& \operatorname{Nat}_{f_{s}}\left(\mathbb{C}^{A}, F\right) \stackrel{\text { def }}{=} \\
& \quad\left\{\alpha \in \Pi_{C \in o b \mathbb{C}}\left|\mathbb{C}(A, C) \Rightarrow_{f_{s}} F C\right| \mid \forall \theta \in \mathbb{C}\left(C, C^{\prime}\right) .(F \theta) \circ \alpha_{C}=\alpha_{C^{\prime}} \circ \theta_{*}\right\}
\end{aligned}
$$


When no confusion can arise, to save space, we write NF for the extensional equality of the (underlying) functions $(F \theta) \circ \alpha_{C}=\alpha_{C^{\prime}} \circ \theta_{*}$ for all $\theta \in \mathbb{C}\left(C, C^{\prime}\right)$. We can then write

$$
\mathrm{Nat}_{e s}\left(\mathbb{C}^{A}, F\right) \stackrel{\text { def }}{=}\left\{\alpha \in \Pi_{C \in o b \mathbb{C}}\left|\left(\mathbb{C}(A, C) \Rightarrow_{f_{s}} F C\right)_{e s}\right| \quad \mid \quad \mathrm{NF}\right\}
$$

The elements of these sets are called (finitely supported/emptily supported = equivariant) ordinary natural families. In the remainder of this section, the isomorphisms in boxes $J \cong K$, and only those, are those that are immediate consequences of the enriched Yoneda Lemmas (Kelly 1982).

Theorem (Weak Yoneda Lemma for $\mathscr{N o m}$ ). Let $\mathbb{C}$ be a Nom-enriched category and $F: \mathbb{C} \rightarrow$ $\mathscr{N}_{0 m}{ }^{e r}$ a Nom-enriched functor. Then there are bijections

$$
(F A)_{e s} \cong \mathscr{S e t}_{\text {et }} \mathscr{N o m}_{-N a t}\left(\mathbb{C}^{A}, F\right) \cong \mathscr{S e t}_{\text {Nat }}\left(\mathbb{C}^{A}, F\right)
$$

Proof. The weak Yoneda Lemma states that $E l(F A) \cong \mathscr{S e t} \mathscr{N}$ om-Nat $\left(\mathbb{C}^{A}, F\right)$. Now in $\mathscr{N}$ om, $E l(X)$ is by definition the set of global elements of $X$, which is the set of emptily supported elements $X_{e s}$. Thus the first bijection holds.

The Nom-natural transformations are (by definition - see the Appendix) families of global elements $\hat{\alpha}=\left(\hat{\alpha}_{C}: 1 \rightarrow \mathscr{N o m}^{e r}(\mathbb{C}(A, C), F C) \mid C \in o b \mathbb{C}\right)$ such that $M \circ\left(\hat{\alpha}_{C^{\prime}} \times \mathbb{C}^{A}\right) \circ \cong_{R}(\theta)=M \circ$ $\left(F \times \hat{\alpha}_{C}\right) \circ \cong_{L}(\theta)$ for all $\theta \in \mathbb{C}\left(C, C^{\prime}\right)$. If one computes each side of the equation, noting that $M$ in $\mathscr{N} \circ \mathrm{m}$ is the regular composition $\circ$, one gets $\hat{\alpha}_{C^{\prime}} \circ \theta_{*}=(F \theta) \circ \hat{\alpha}_{C}$. Since the families of global elements $\hat{\alpha}$ are trivially in bijection with $\left(\alpha_{C} \in\left(\mathbb{C}(A, C) \Rightarrow_{f_{s}} F C\right)_{e s} \mid C \in o b \mathbb{C}\right)$, the second bijection holds.

Theorem (Weak Yoneda Lemma for $\mathscr{F} \mathscr{M} \mathscr{N}$ om and $\mathscr{F} \mathscr{M} \mathscr{S}$ et). Let $\mathbb{C}$ be a $\mathscr{V}$-enriched category and $F: \mathbb{C} \rightarrow \mathscr{V}^{e r}$ a $\mathscr{V}$-enriched functor where $\mathscr{V}$ is either $\mathscr{F} \mathscr{M} \mathscr{N}$ om or $\mathscr{F} \mathscr{M} \mathscr{S e t}$. Then there are bijections

$$
F A \cong \mathscr{S e t}_{\mathrm{V}} \mathscr{V} \operatorname{Nat}\left(\mathbb{C}^{A}, F\right) \cong \operatorname{Set}_{\mathrm{Nat}} \mathrm{fs}_{\mathrm{s}}\left(\mathbb{C}^{A}, F\right)
$$

Proof. In each $\mathscr{V}, E l(X)$ is the whole of $X$. The result follows by computations very similar to those of Theorem 3.1.

\section{The Strong Yoneda Lemma}

To try to assist the reader, the meanings of symbols $\mathbb{C}, \mathbb{D}, S$ and $T$ are defined in Section A.1. In this section, unless stated otherwise, we take $\mathbb{C}$ any $\mathscr{V} \stackrel{\text { def }}{=} \mathscr{N}$ om-enriched category, $\mathbb{D} \stackrel{\text { def }}{=} \mathscr{N} o m^{e r}$, $S \stackrel{\text { def }}{=} \mathbb{C}^{A}: \mathbb{C} \rightarrow \mathscr{N} m^{\text {er }}$ and $T \stackrel{\text { def }}{=} F: \mathbb{C} \rightarrow \mathscr{N} m^{\text {er }}$ any $\mathscr{N}$ om-functor. We then have

$$
\left[\mathbb{C}, \mathscr{N} o m^{e r}\right]\left(\mathbb{C}^{A}, F\right) \stackrel{\text { def }}{=} \int_{C \in o b \mathbb{C}} \mathscr{N} m^{e r}(\mathbb{C}(A, C), F C)=\int_{C \in o b \mathbb{C}} \mathbb{C}(A, C) \Rightarrow_{f s} F C
$$

with the second equality following from the definition of $\mathscr{N}_{0} m^{e r}$. Note that we write $i d_{A} \stackrel{\text { def }}{=} j_{A}(*) \in$ $\mathbb{C}(A, A)$; of course $i d_{A}$ is emptily supported.

Theorem (Strong Yoneda Lemma for Nom).

$$
F A \cong \mathscr{N o m}\left[\mathbb{C}, \mathscr{N}_{o m}{ }^{e r}\right]\left(\mathbb{C}^{A}, F\right)=\operatorname{Nat}_{f s}\left(\mathbb{C}^{A}, F\right)
$$

with permutation action on $\alpha \in\left[\mathbb{C}, \mathscr{N}_{o m}{ }^{e r}\right]\left(\mathbb{C}^{A}, F\right)$ given by $\left(\pi \cdot \int \alpha\right)_{C}=\pi \cdot \Rightarrow_{f s} \alpha_{C}$. 
Proof. The end instance is the $\mathscr{N}$ om equaliser $\operatorname{EQR}(\rho, \sigma)$ of the Nom diagram

$$
\Pi_{C \in o b} \mathbb{C} \mathbb{C}(A, C) \Rightarrow_{f_{s}} F C \underset{\sigma}{\stackrel{\rho}{\longrightarrow}} \Pi_{C, C^{\prime} \in o b \mathbb{C}} \mathbb{C}\left(C, C^{\prime}\right) \Rightarrow_{f_{s}}\left(\mathbb{C}(A, C) \Rightarrow_{f_{s}} F C^{\prime}\right)
$$

where, following the Appendix page 1023, we obtain

$$
\begin{array}{cc}
\sigma \stackrel{\text { def }}{=}\left\langle\sigma_{C, C^{\prime}} \circ p r_{C^{\prime}}\right\rangle \quad \sigma_{C, C^{\prime}}=\lambda\left(M_{\mathbb{C}(A, C), \mathbb{C}\left(A, C^{\prime}\right), F C^{\prime}} \circ\left(i d_{\mathbb{C}\left(A, C^{\prime}\right) \Rightarrow_{f_{s}} F C^{\prime}} \times \mathbb{C}_{C, C^{\prime}}^{A}\right)\right) \\
\rho \stackrel{\text { def }}{=}\left\langle\rho_{C, C^{\prime}} \circ p r_{C}\right\rangle \quad \rho_{C, C^{\prime}}=\lambda\left(M_{\mathbb{C}(A, C), F C, F C^{\prime}} \circ\left(F_{C, C^{\prime}} \times i d_{\mathbb{C}(A, C) \Rightarrow_{f_{s}} F C}\right) \stackrel{\cong}{=}\right)
\end{array}
$$

If $\alpha \in \Pi_{C \in o b} \mathbb{C} \mathbb{C}(A, C) \Rightarrow_{f_{s}} F C$ and $\theta \in \mathbb{C}\left(C, C^{\prime}\right)$, by calculating with the definitions we see that the equaliser requirement $\rho(\alpha)=\sigma(\alpha)$ also amounts to NF. Now, working in $\mathscr{N}$ om, the product $\Pi_{C \in o b \mathbb{C}} \mathbb{C}(A, C) \Rightarrow_{f s} F C$ is

$$
\left\{\left(\alpha_{C} \in \mathbb{C}(A, C) \Rightarrow_{f s} F C \mid C \in o b \mathbb{C}\right) \mid \bigcup_{C \in o b \mathbb{C}} \operatorname{supp}\left(\alpha_{C}\right)<\infty\right\}
$$

and the permutation action is given by $(\pi \cdot \alpha)_{C}=\pi \cdot \Rightarrow_{f s} \alpha_{C}$. Hence

$$
\left[\mathbb{C}, \mathscr{N}_{o m}{ }^{\text {er }}\right]\left(\mathbb{C}^{A}, F\right)=\operatorname{EQR}(\rho, \sigma) \in o b \mathscr{N o m}
$$

is

$$
\left\{\left(\alpha_{C} \in \mathbb{C}(A, C) \Rightarrow_{f_{s}} F C \mid C \in o b \mathbb{C}\right) \mid \bigcup_{C \in o b \mathbb{C}} \operatorname{supp}\left(\alpha_{C}\right)<\infty \wedge \mathrm{NF}\right\}
$$

with $\left(\pi \cdot \int \alpha\right)_{C}=\pi \cdot \Rightarrow_{f_{s}} \alpha_{C}$. It is immediate that

$$
\left[\mathbb{C}, \mathscr{N}_{o m}{ }^{\text {er }}\right]\left(\mathbb{C}^{A}, F\right) \subseteq \operatorname{Nat}_{f s}\left(\mathbb{C}^{A}, F\right)
$$

We claim that this is in fact an equality. Choose any $\alpha \in N a t_{f s}\left(\mathbb{C}^{A}, F\right)$. If we can show supp $\left(\alpha_{C}\right) \subseteq$ $\operatorname{supp}\left(\alpha_{A}\left(i d_{A}\right)\right)$, it is then trivial that

$$
\bigcup_{C \in o b \mathbb{C}} \operatorname{supp}\left(\alpha_{C}\right) \subseteq \operatorname{supp}\left(\alpha_{A}\left(i d_{A}\right)\right)<\infty,
$$

and the proof is complete. Using an instance of NF with $C \stackrel{\text { def }}{=} A$ and $C^{\prime} \stackrel{\text { def }}{=} C$ and hence $\theta \in \mathbb{C}(A, C)$ we get

$$
(F \theta)\left(\alpha_{A}\left(i d_{A}\right)\right)=\left(F \theta \circ \alpha_{A}\right)\left(i d_{A}\right)=\left(\alpha_{C} \circ \theta_{*}\right)\left(i d_{A}\right)=\alpha_{C}\left(\theta \circ i d_{A}\right)=\alpha_{C}(\theta)
$$

Hence if $\tau \# \alpha_{A}\left(i d_{A}\right)$, noting $\tau \cdot \theta \in \mathbb{C}(A, C)$ we have

$$
\begin{aligned}
\left(\tau \cdot \alpha_{C}\right)(\theta) & =\tau \cdot\left(\alpha_{C}(\tau \cdot \theta)\right) \\
& =\tau \cdot\left(F_{A, C}(\tau \cdot \theta)\left(\alpha_{A}\left(i d_{A}\right)\right)\right) \\
& =\tau \cdot\left(\left(\tau \cdot\left(F_{A, C} \theta\right)\right)\left(\alpha_{A}\left(i d_{A}\right)\right)\right) \\
& =\left(F_{A, C} \theta\right)\left(\tau \cdot\left(\alpha_{A}\left(i d_{A}\right)\right)\right) \\
& =\left(F_{A, C} \theta\right)\left(\alpha_{A}\left(i d_{A}\right)\right) \\
& =\alpha_{C}(\theta) .
\end{aligned}
$$

Equations (1) and (4) are trivial properties of any perm action; (2) and (6) are by instances of NF; (5) is from the freshness hypothesis; and (3) holds because $F_{A, C}: \mathbb{C}(A, C) \rightarrow F A \Rightarrow_{f s} F C$ is equivariant.

We further examine the strong Yoneda Lemma in Theorem 4, looking in detail at the structures of the underlying sets. 
Theorem (Strong Yoneda: Bare Hands Version). If $|F A|$ is the underlying set of $F A$, there is a setbijection $\Phi:|F A| \cong \mathscr{S}_{\text {et }}\left|N a t_{f s}\left(\mathbb{C}^{A}, F\right)\right|: \Psi$ given by $\Psi(\alpha) \stackrel{\text { def }}{=} \alpha_{A}\left(i d_{A}\right)$ and $\Phi(x) \stackrel{\text { def }}{=} \bar{x}$ with $\bar{x}_{C}(\theta) \stackrel{\text { def }}{=}$ $(F \theta)(x)$. Moreover $\left|\mathrm{Nat}_{f s}\left(\mathbb{C}^{A}, F\right)\right|$ becomes a nominal set with permutation action $*$ defined by passing the action of FA across the bijection, that is $\pi * \alpha \stackrel{\text { def }}{=} \Phi(\pi \cdot F A \Psi(\alpha))$. Moreover the permutation action from Theorem 4 coincides with this action, that is $\pi \cdot \int \alpha=\pi * \alpha$, leading to an isomorphism of nominal sets in Nom which is natural in $A$ and $F$

$$
\left.\Phi: F A \cong \mathscr{N o m}_{\left(N a t_{f s}\right.}\left(\mathbb{C}^{A}, F\right) \mid, *\right)=\operatorname{Nat}_{f s}\left(\mathbb{C}^{A}, F\right): \Psi .
$$

Proof. The $\mathscr{S e t}$ bijection is the standard one, hence $\operatorname{NF}(\bar{x})$ holds provided that each $\bar{x}$ is finitely supported. Let $\tau \# x \in F A$. Then for any $\theta \in \mathbb{C}(A, C)$

$$
\begin{aligned}
\left(\tau \Rightarrow_{f_{s}} \bar{x}_{C}\right)(\theta) & =\tau \cdot \cdot_{F C}\left(\bar{x}_{C}(\tau \cdot \mathbb{C}(A, C) \theta)\right) \\
& =\tau \cdot F C \\
& \left.=\tau \cdot F_{A, C}(\tau \cdot \mathbb{C}(A, C) \theta)(x)\right) \\
& \left.\left.=\tau \cdot \Rightarrow_{f s}\left(F_{A, C} \theta\right)\right)(x)\right) \\
& =\left(F_{A, C} \theta\right)(x) \\
& \stackrel{\text { def }}{=} \bar{x}_{C}(\theta) .
\end{aligned}
$$

The calculation is easy, again using the fact that $F_{A, C}: \mathbb{C}(A, C) \rightarrow F A \Rightarrow_{f s} F C$ is equivariant. Next, $\pi \cdot \int \alpha=\pi * \alpha$ also follows from a calculation:

$$
\begin{aligned}
(\pi * \alpha)_{C}(\theta) & =\Phi(\pi \cdot F A \Psi(\alpha))(\theta) \\
& \left.=\overline{(\pi \cdot F A} \alpha_{A}\left(i d_{A}\right)\right)_{C}(\theta) \\
& =(F \theta)\left(\pi \cdot F A \alpha_{A}\left(i d_{A}\right)\right) \\
& =\pi \cdot F C \pi^{-1} \cdot F C(F \theta)\left(\pi \cdot F A \alpha_{A}\left(i d_{A}\right)\right) \\
& =\pi \cdot F C\left(\left(\pi^{-1} \cdot F C(F \theta)\right)\left(\alpha_{A}\left(i d_{A}\right)\right)\right) \\
& =\pi \cdot F C\left(F\left(\pi^{-1} \cdot \mathbb{C}(A, C) \theta\right)\left(\alpha_{A}\left(i d_{A}\right)\right)\right) \\
& =\pi \cdot F C \alpha_{C}\left(\pi^{-1} \cdot \mathbb{C}(A, C) \theta\right) \\
& =\left(\pi \cdot \int \alpha\right)(\theta) .
\end{aligned}
$$

That $\Phi$ and $\Psi$ are equivariant, and the $\mathscr{N} o m$ isomorphism, follows immediately from $\cdot \rho=*$ and Lemma 1.

\section{Variations of the Yoneda Lemmas}

We can deduce the following interesting corollary from the previous results, where ordinary natural families coincide with finitely supported natural families.

Corollary 5. Let $\mathbb{C}$ be a Nom-enriched category and F: $\mathbb{C} \rightarrow \mathscr{N}^{\text {er }}{ }^{\text {er }}$ a Nom-enriched functor. For $A \in o b \mathbb{C}$ if we define

$$
\operatorname{Nat}\left(\mathbb{C}^{A}, F\right) \stackrel{\text { def }}{=}\left(\left\{\alpha \in \Pi_{C \in o b} \mathbb{C}(|\mathbb{C}(A, C)| \Rightarrow|F C|) \mid \mathrm{NF}\right\}, \cdot \int\right)
$$

then we have

$$
\left|\operatorname{Nat}\left(\mathbb{C}^{A}, F\right)\right|=\mathscr{S e t}_{\text {et }}\left|\operatorname{Nat}_{f_{s}}\left(\mathbb{C}^{A}, F\right)\right|
$$


that is, all such natural transformations are finitely supported; and moreover

$$
F A \cong N_{N o m} \operatorname{Nat}\left(\mathbb{C}^{A}, F\right)
$$

This result follows by combining previous results with the Yoneda Lemma over $\mathscr{S} e t=\mathscr{S} e t^{e r}$ and a 'localised' forgetful functor. We give the definitions. Let $\mathbb{C}$ be $\mathscr{N} o m$-enriched. The $(\mathscr{S}$ etenriched) category ${ }^{1} U \mathbb{C}$ has objects those of $\mathbb{C},(U \mathbb{C})(A, B) \stackrel{\text { def }}{=}|\mathbb{C}(A, B)|$, identity $J_{A}^{U \mathbb{C}}: 1 \rightarrow$ $(U \mathbb{C})(A, A)=|\mathbb{C}(A, A)|$ given by $* \mapsto j_{A}^{\mathbb{C}}(*)$, and (similarly) $M^{U \mathbb{C}}$ is the set-theoretic function composition underlying $M^{\mathbb{C}}$. The functor $U F: U \mathbb{C} \rightarrow \mathscr{S e t} t^{e r}$ is defined by $(U F)(A) \stackrel{\text { def }}{=}|F A|$ and $(U F)_{A, B}:|\mathbb{C}(A, B)| \rightarrow \operatorname{Set}(F A, F B)=|F A| \Rightarrow|F B|$ is defined by

$$
f \mapsto F_{A, B}(f) \in\left|F A \Rightarrow_{f_{s}} F B\right| \stackrel{\text { def }}{=}(|F A| \Rightarrow|F B|)_{f_{s}} \subseteq|F A| \Rightarrow|F B| .
$$

Now we can give the proof.

Proof. We have $(U F)(A) \cong \mathscr{S e t}\left[\mathbb{C}, \mathscr{S} e t^{e r}\right]\left((U \mathbb{C})^{A}, U F\right)$ from the $\mathscr{S e t}^{\text {er }}$ strong Yoneda Lemma where the RHS is the equaliser

$$
E Q R(\rho, \sigma) \stackrel{\text { def }}{=}\left\{\left(\alpha_{C} \in(U \mathbb{C})(A, C) \Rightarrow(U F) C \mid C \in o b \mathbb{C}\right) \mid \mathrm{NF}\right\}
$$

which is precisely $\left|\operatorname{Nat}\left(\mathbb{C}^{A}, F\right)\right|$. From Theorem $4 F A \cong \mathscr{N o m}_{N a t}\left(\mathbb{C}^{A}, F\right)$. Hence by Lemma 1 we have $\left|\operatorname{Nat}\left(\mathbb{C}^{A}, F\right)\right| \cong \mathscr{S e t}_{\text {et }}\left|\operatorname{Nat}_{f s}\left(\mathbb{C}^{A}, F\right)\right|$, and if one computes the (Yoneda) bijection explicitly, one sees that it is the identity. It follows from Lemma 1 that $F A \cong \mathscr{N o m}_{N} \operatorname{Nat}\left(\mathbb{C}^{A}, F\right)$.

The following result, for ordinary categories $\mathscr{C}$, is also a corollary of the work so far.

Theorem (Ordinary Nominal Yoneda Lemma). Suppose that $\mathscr{C}$ is an ordinary category, and that $\mathscr{V}$ is either $\mathscr{N o m}$ or $\mathscr{F} \mathscr{M} \mathscr{N}$ om. There is a trivial enrichment of $\mathscr{C}$ over each $\mathscr{V}$, taking discrete hom-sets. Writing $\mathscr{V}^{\mathscr{C}}$ for the ordinary functor category, and taking $F \in o b \mathscr{V}^{\mathscr{C}}$, we have

$$
(F A)_{e s} \cong \mathscr{S e t}_{e} \mathscr{N}_{o m} \mathscr{C}\left(\mathscr{C}^{A}, F\right) \quad F A \cong \mathscr{L e t}_{\mathscr{F}} \mathscr{M} \mathscr{N}_{o m} \mathscr{C}\left(\mathscr{C}^{A}, F\right)
$$

Further, let the category $\mathscr{F} \mathscr{M} \mathscr{N}_{\text {omer }}^{\mathscr{C}}$ consist of objects those functors $F$ that are pseudo- $N$ omenriched, by which we mean the morphism actions $F_{C, C^{\prime}}: \mathscr{C}\left(C, C^{\prime}\right) \rightarrow F C \Rightarrow_{f_{s}} F C^{\prime}$ are equivariant.

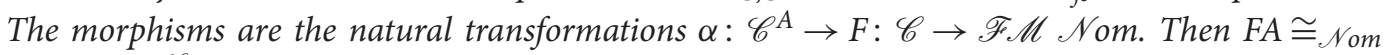
$\mathscr{F} \mathscr{M} \mathscr{N}_{\text {omer }}^{\mathscr{C}}\left(\mathscr{C}^{A}, F\right)$.

Proof. This result follows from instances of Theorem 3.1 for the first two bijections, and Theorem 4 for the second, since ordinary natural transformations are ordinary natural families.

We now present an internal Yoneda Lemma for $\mathscr{F} \mathscr{M} \mathscr{N}$ om and $\mathscr{F} \mathscr{M} \mathscr{S e t}$ (see page Section A.2 for a notation summary). We write $\mathscr{V}$ for either of these categories: since $\mathscr{F} \mathscr{M} \mathscr{N}$ om is a full subcategory of $\mathscr{F} \mathscr{M} \mathscr{S e t}$, the key proof details are similar in both cases (much the same in spirit as Theorem 3.1).

Let $\mathbb{C}$ be an internal category in $\mathscr{V}$ with structure morphisms $\left(d_{0}, d_{1}, \iota, c\right)$ for source, target, identities and composition (where, for example, $d_{0}: \mathbb{C}_{1} \rightarrow \mathbb{C}_{0}$ and so on). Then the internal $\mathscr{V}$ valued hom-functor $Y^{A}: \mathbb{C} \rightarrow \mathscr{V}$ for $A \in \mathbb{C}_{0}$ is specified by $Y^{A} \stackrel{\text { def }}{=}\left(d_{0}^{-1}(A), \hat{d}_{1}, \hat{c}\right)$ where

$$
\hat{d}_{1} \stackrel{\text { def }}{=} d_{\left.1\right|_{d_{0}^{-1}(A)}}: d_{0}^{-1}(A) \rightarrow \mathbb{C}_{0} \quad \hat{c} \stackrel{\text { def }}{=} c_{\mathbb{C}_{1} \times \mathbb{C}_{0} d_{0}^{-1}(A)}: \mathbb{C}_{1} \times \mathbb{C}_{0} d_{0}^{-1}(A) \rightarrow d_{0}^{-1}(A) .
$$

This is a good definition: the morphisms are finitely supported by $\operatorname{supp}\left(d_{1}\right)$ and $\operatorname{supp}(c)$ respectively. The axioms for $Y^{A}$ to be an $\mathscr{V}$-valued functor follow trivially: for example, $\hat{d}_{1} \circ \hat{c}=d_{1} \circ p r_{\mathbb{C}_{1}}$ is immediate since $d_{1} \circ c=d_{1} \circ p r_{\mathbb{C}_{1}}$ in $\mathbb{C}$. The internal functor category $\mathscr{V} \mathbb{C}$ follows the usual definition. 
Theorem (Internal Yoneda Lemma for $\mathscr{V}$ ). If $\mathbb{C}$ is an internal category in $\mathscr{V}$ and $F: \mathbb{C} \rightarrow \mathscr{V}$ is an $\mathscr{V}$-valued internal functor, then for $A \in \mathbb{C}_{0}$ we have

$$
\Phi: F A \cong \mathscr{V} \mathscr{V}^{\mathbb{C}}\left(\mathrm{Y}^{A}, F\right),: \Psi
$$

where $F \stackrel{\text { def }}{=}\left(Q, q_{0}, q_{1}\right)$ and $F A \stackrel{\text { def }}{=} q_{0}^{-1}(A)$. If $\alpha: Y^{A} \rightarrow F$ is specified by some finitely supported function $\alpha: d_{0}^{-1}(A) \rightarrow Q$ then we put $\Psi(\alpha) \stackrel{\text { def }}{=} \alpha(\iota(A))$ and $\Phi(x \in F A): d_{0}^{-1}(A) \rightarrow Q$ where $\Phi(x)(f) \stackrel{\text { def }}{=}$ $q_{1}(f, x)$.

Proof. The verification of the theorem is a lengthy calculation, most of which we omit. We examine one small part of the proof. Let us show that $\Phi$ is a morphism in $\mathscr{V}$, that is, it has finite support, namely $\operatorname{supp}\left(q_{1}\right)$. Choose $\tau \# q_{1}$ and pick $x \in \tau \cdot F A$. Then, since $\tau \cdot q_{1}=q_{1}$, and $\tau \cdot \tau \cdot \xi=\xi$ for any $\xi$,

$$
(\tau \cdot \Phi)(x)(f)=(\tau \cdot(\phi(\tau \cdot x)))(f)=\tau \cdot[(\phi(\tau \cdot x))(\tau \cdot f)]=\tau \cdot\left[q_{1}(\tau \cdot f, \tau \cdot x)\right]=\Phi(x)(f) .
$$

\section{Cartesian Closure of Functor Categories}

The following theorem is interesting in its own right, building up our understanding of 'nominal functor categories'. In particular, it is the cartesian closure of the category $\mathscr{F} \mathscr{M} \mathscr{N}_{\text {om }}^{\mathscr{C}}$ ner (defined in Theorem 5) that will play an important role in FM gluing, analogous to that of $\mathscr{S e t} t^{\mathscr{C}}$ and $\omega \mathscr{C} \mathscr{P} \mathscr{O}^{\mathscr{C}}$ in Crole (1996). We plan for this to appear in a future paper.

Theorem. The categories $\mathscr{N}_{0} \mathscr{C}$ and $\mathscr{F} \mathscr{M} \mathscr{N}_{0}^{\mathscr{C}}$ ner are both cartesian closed.

Proof. We give details for $\mathscr{F} \mathscr{M} \mathscr{N}_{\text {om }}^{\mathscr{C}}$ ner t the proof for $\mathscr{N}^{\mathscr{C}} \mathbb{C}^{\mathscr{C}}$ is very similar. Let us write $\mathscr{F}$ for $\mathscr{F} \mathscr{M} \mathscr{N}_{0} m_{\text {ner }}^{\mathscr{C}}$. Crucially, since we have proved that $F A \cong \mathscr{N}_{\mathrm{om}} \mathscr{F}\left(\mathscr{C}^{A}, F\right)$, we can use this to construct exponentials - in the same way as one proceeds in presheaf categories. Take any $F, F^{\prime} \in o b \mathscr{F}$. If the exponential exists then we must have $\left(F \Rightarrow F^{\prime}\right)(A) \cong \mathscr{N o m}_{\mathscr{F}}\left(\mathscr{C}^{A}, F \Rightarrow\right.$ $\left.F^{\prime}\right) \cong \mathscr{N}_{\text {om }} \mathscr{F}\left(\mathscr{C}^{A} \times F, F^{\prime}\right)$ from Theorem 5 . This guides us to set

$$
\begin{aligned}
(F \Rightarrow & \left.F^{\prime}\right)(A) \stackrel{\text { def }}{=} \\
& \mathscr{F}\left(\mathscr{C}^{A} \times F, F^{\prime}\right) \stackrel{\text { def }}{=}\left\{\gamma \in \Pi_{C \in o b \mathscr{C}} \mathscr{C}(A, C) \times F C \Rightarrow_{f_{s}} F^{\prime} C \mathrm{NF}(\gamma)\right\}
\end{aligned}
$$

and if $a: A \rightarrow A^{\prime}$ in $\mathscr{C}$ then $\left(F \Rightarrow F^{\prime}\right)(a):\left(\gamma_{C} \mid C\right) \mapsto\left(\gamma_{C} \circ\left(a^{*} \times i d_{F C}\right) \mid C\right)$. One can check that $\left(F \Rightarrow F^{\prime}\right)(A)$ is a nominal set with $\left(\pi \cdot \int \gamma\right)_{C}=\pi \Rightarrow_{f_{s}} \gamma_{C}$ where $\operatorname{supp}(\gamma)=\operatorname{supp}\left(\gamma_{A}\right)$. Observing the definition of $\mathscr{F}$ we need to check that $\pi \cdot\left(F \Rightarrow F^{\prime}\right)(a)=\left(F \Rightarrow F^{\prime}\right)(a)$. Now, note in general that if $\theta: C \rightarrow C^{\prime}$, then the requirement that $G_{C, C^{\prime}}(\theta)=\pi \cdot G_{C, C^{\prime}}(\theta)$ holds just in case $G_{C, C^{\prime}}(\theta)$ is itself equivariant. Here, it is easiest to verify the equivariance of $\left(F \Rightarrow F^{\prime}\right)(a)$. We have, since $a^{*} \times i d_{F C}$ is an equivariant function,

$$
\begin{aligned}
{\left[\left(F \Rightarrow F^{\prime}\right)(a)\left(\pi \cdot \int \gamma\right)\right]_{C} } & =\left(\pi \cdot \int \gamma\right)_{C} \circ\left(a^{*} \times i d_{F C}\right) \\
& =\left(\pi \cdot \Rightarrow_{f_{s}} \gamma_{C}\right) \circ\left(a^{*} \times i d_{F C}\right) \\
& =\left(\pi \cdot \Rightarrow_{f_{s}} \gamma_{C}\right) \circ\left(\pi \cdot\left(a^{*} \times i d_{F C}\right)\right) \\
& =\pi \cdot \Rightarrow_{f s}\left(\gamma_{C} \circ\left(a^{*} \times i d_{F C}\right)\right) \\
& =\left[\pi \cdot \int\left(F \Rightarrow F^{\prime}\right)(a)(\gamma)\right]_{C} .
\end{aligned}
$$

The specifications of the bijections are analogous to those for presheaf toposes. Given $\left(\alpha_{A}: G A \times\right.$ $\left.F A \rightarrow F^{\prime} A \mid A\right)$ then $\left((\lambda \alpha)_{A}: G A \rightarrow\left(F \Rightarrow F^{\prime}\right)(A) \mid A\right)$ is defined by

$$
\left((\lambda \alpha)_{A}(x)\right)_{C}:(\theta, y) \mapsto \alpha_{C}((G \theta)(x), y)
$$


and for $\left(\beta_{A}: G A \rightarrow\left(F \Rightarrow F^{\prime}\right)(A) \mid A\right)$ then $\left(\bar{\beta}_{A}: G A \times F A \rightarrow F^{\prime} A \mid A\right)$ is defined by

$$
\bar{\beta}_{A}:(x, y) \mapsto\left(\beta_{A}(x)\right)_{A}\left(i d_{A}, y\right) .
$$

The naturality properties hold with the proofs mimicking those in a presheaf topos (Johnstone 1977); the same is true of the computations that $\overline{\lambda \alpha}=\alpha$ and that $\lambda \bar{\beta}=\beta$. However, we do need to make sure the bijections are well defined, and this we do in some detail for $\alpha$, verifying the support properties. We show, below, that each $(\lambda \alpha)_{A}$ is finitely supported (by $\left.\operatorname{supp}\left(\alpha_{A}\right)\right)$; and that each $\left((\lambda \alpha)_{A}(x)\right)_{C}$ is finitely supported (by $\left.\operatorname{supp}\left(\alpha_{A}\right) \cup \operatorname{supp}(x)\right)$. We leave the proof of $\operatorname{supp}\left(\bar{\beta}_{A}\right)=$ $\operatorname{supp}\left(\beta_{A}\right)$ to the reader. Choose $\tau \# \alpha_{A}$. Then

$$
\begin{aligned}
\left(\left(\tau \cdot(\lambda \alpha)_{A}\right)(x)\right)_{C}(\theta, y) & =\left(\tau \cdot\left((\lambda \alpha)_{A}(\tau \cdot x)\right)\right)_{C}(\theta, y) \\
& =\tau \cdot\left(\left((\lambda \alpha)_{A}(\tau \cdot x)\right)\right)_{C}(\theta, \tau \cdot y) \\
& =\tau \cdot\left(\alpha_{C}((G \theta)(\tau \cdot x), \tau \cdot y)\right) \\
& =\left(\tau \cdot \alpha_{C}\right)((\tau \cdot(G \theta))(x), y) \\
& =\left(\alpha_{C}\right)((G \theta)(x), y) \\
& =\left(\left((\lambda \alpha)_{A}(x)\right)_{C}\right)(\theta, y) .
\end{aligned}
$$

Choose $\tau \# \alpha_{A}, x$. Then

$$
\begin{aligned}
\left(\tau \cdot\left((\lambda \alpha)_{A}(x)\right)_{C}\right)(\theta, y) & =\tau \cdot\left((\lambda \alpha)_{A}(x)\right)_{C}(\theta, \tau \cdot y) \\
& =\tau \cdot \alpha_{C}((G \theta)(x), \tau \cdot y) \\
& =\left(\tau \cdot \alpha_{C}\right)(\tau \cdot[(G \theta)(x)], y) \\
& =\alpha_{C}((G \theta)(\tau \cdot x), y) \\
& =\alpha_{C}((G \theta)(x), y) \\
& =\left((\lambda \alpha)_{A}(x)\right)_{C}(\theta, y)
\end{aligned}
$$

\section{Conclusions and Further Work}

This paper grew from plans to use categorical gluing to establish the conservativity of pure NLC over NEL. In the early stages of this work, we realised that some of the categories that we might need to glue over were somewhat troublesome in that there was a lack of (co)completeness. We needed to verify cartesian closure of (some of) the categories and this led to our analysis of the Yoneda Lemma.

The computations in prelimary work are very 'fine grained' and so as a consequence we wanted to understand and make use of the FM Yoneda results not just abstractly, but concretely in the forms presented in this paper where we can compute explicitly with the permutation actions. Thus Theorem 4 is especially useful.

\section{Note}

1 Note: $U \mathbb{C}$ is not $\mathbb{C}_{0}$ !

\section{References}

Borceux, F. (1994a). Handbook of Categorical Algebra: Volume 1, Basic Category Theory, Encyclopedia of Mathematics and its Applications, Cambridge, Cambridge University Press. https://doi.org/10.1017/CBO9780511525858.

Borceux, F. (1994b). Handbook of Categorical Algebra: Volume 2, Categories and Structures, Encyclopedia of Mathematics and its Applications, Cambridge, Cambridge University Press. https://doi.org/10.1017/CBO9780511525865.

Carboni, A. and Johnstone, P. (1995). Connected limits, familial representability and artin glueing. Mathematical Structures in Computer Science 5 (4) 441-459. 
Clouston, R. (2011). Nominal Lawvere theories. In: WoLLIC'11, 67-83.

Clouston, R. (2014). Nominal Lawvere theories: A category theoretic account of equational theories with names. Journal of Computer and System Sciences 80 (6), 1067-1086.

Clouston, R. and Pitts, A. M. (2007). Nominal equational logic. Electronic Notes in Theoretical Computer Science 172 $223-257$.

Crole, R. L. (1993). Categories for Types, Cambridge, Cambridge University Press.

Crole, R. L. (1996). On fixpoint objects and gluing constructions. Applied Categorical Structures 4 (2 \& 3), 251-281. This volume is a Special Edition for the European Colloquium on Category Theory, Tours, France.

Crole, R. L. and Nebel, F. (2013). Nominal lambda calculus: An internal language for FM-cartesian closed categories. In: Mislove, M. (ed.), Proceedings of Twenty-Ninth Conference on the Mathematical Foundations of Programming Semantics (MFPS XXIX), New Orleans, LA, Electronic Notes in Theoretical Computer Science, vol. 298, 93-117.

Gabbay, M. and Pitts, A. M. (2002). A new approach to abstract syntax with variable binding. Formal Aspects of Computing $13(3-5)$ 341-363.

Gabbay, M. J. A Theory of Inductive Definitions with alpha-Equivalence.

Gabbay, M. J. and Mathijssen, A. (2009). Nominal universal algebra: Equational logic with names and binding. Journal of Logic and Computation 19 (6) 1455-1508.

Johnstone, P. (1977). Topos Theory, LMS Monographs, Cambridge, MA, Academic Press.

Johnstone, P. T. (2002). Sketches of an Elephant: A Topos Theory Compendium, Oxford Logic Guides, Oxford, Oxford University Press.

Kelly, G. (1982). Basic Concepts of Enriched Category Theory, London Mathematical Society Lecture Note Series, vol. 64, Cambridge, Cambridge University Press.

Kelly, G. (2005). Basic Concepts of Enriched Category Theory. Reprints in Theory and Applications of Categories, No. 10. Lambek, J. and Scott, P. (1986). Introduction to Higher Order Categorical Logic, Cambridge, Cambridge University Press. Lane, S. (1998). Categories for the Working Mathematician, Graduate Texts in Mathematics, Berlin, Heidelberg, Springer. Moerdijk, I. (1982). Glueing topoi and higher order disjunction and existence. In: Troelstra, A. S. and van Dalen, D. (eds.), The L. E. J. Brouwer Centenary Symposium, Studies in Logic and the Foundation of Mathematics, North-Holland, $359-375$.

Mostowski, A. (1939). Uber die Unabhangigkeit des Wohlordnungssatzes vom Ordnungsprinzip. Fundamenta Mathematicae 32 201-252.

Pitts, A. M. (2003). Nominal logic, a first order theory of names and binding. Information and Computation 186 165-193.

Pitts, A. M. (2013). Nominal Sets: Names and Symmetry in Computer Science, Cambridge Tracts in Theoretical Computer Science, vol. 57, Cambridge, Cambridge University Press.

\section{Appendix A. Category Theoretic Notation}

\section{A.1 Enriched category theory}

We follow Kelly (1982) with some mild notational changes. Let $\mathscr{V}$ be a cartesian closed category. Recall that a category $\mathbb{C}$ enriched over $\mathscr{V}$ (or a $\mathscr{V}$-category) has: a collection of objects, $o b \mathbb{C}$; if $A$ and $B$ are objects a morphism object $\mathbb{C}(A, B) \in o b \mathbb{C}$; identities $j_{A}: 1 \rightarrow \mathbb{C}(A, A)$ each a morphism in $\mathscr{V}$; and composition morphisms $M_{A, B, C}: \mathbb{C}(B, C) \times \mathbb{C}(A, B) \rightarrow \mathbb{C}(A, C)$ in $\mathscr{V}$ all satisfying the usual equations. A $\mathscr{V}$-functor $S: \mathbb{C} \rightarrow \mathbb{D}$ is specified by a mapping $S: o b \mathbb{C} \rightarrow o b \mathbb{D}$ and a morphism $S_{A, B}: \mathbb{C}(A, B) \rightarrow \mathbb{D}(S A, S B)$ in $\mathscr{V}$ subject to the standard equations. A $\mathscr{V}$-natural transformation $\alpha: S \rightarrow T: \mathbb{C} \rightarrow \mathbb{D}$ is a family $\left(\alpha_{C}: 1 \rightarrow \mathbb{D}(S C, T C) \mid C \in o b \mathbb{C}\right)$ of global elements in $\mathscr{V}$ such that $M \circ\left(\alpha_{C}^{\prime} \times S\right) \circ \cong_{R}=M \circ\left(T \times \alpha_{C}\right) \circ \cong_{L}$ in $\mathscr{V}$ where

$$
\mathbb{C}\left(C, C^{\prime}\right) \stackrel{\cong_{R}}{\longrightarrow} 1 \times \mathbb{C}\left(C, C^{\prime}\right) \quad \mathbb{C}\left(C, C^{\prime}\right) \stackrel{\cong_{L}}{\longrightarrow} \mathbb{C}\left(C, C^{\prime}\right) \times 1
$$

We write $\mathscr{V}-\operatorname{Nat}(S, T)$ for the collection of such $\mathscr{V}$-natural transformations. If $\mathscr{V}$ is locally small, the elements functor is $E l(+) \stackrel{\text { def }}{=} \mathscr{V}(1,+): \mathscr{V} \rightarrow \mathscr{S e t}$.

Suppose that $\mathscr{V}$ is a ccc. Write $\mathscr{V}^{e r}$ for the enrichment of “ $\mathscr{V}$ over itself”, so that $\mathscr{V}^{e r}(A, B) \stackrel{\text { def }}{=}$ $A \Rightarrow B$ is the $\mathscr{V}$ exponential, and $j_{A}$ and $M_{A, B, C}$ are given the usual definitions as exponential mates (eg if $p r: 1 \times A \rightarrow A$ in $\mathscr{V}$ then $j_{A} \stackrel{\text { def }}{=} \lambda p r$ ). Note that Kelly writes $[A, B]$ for our $A \Rightarrow B$; we prefer the latter since the notation $\Rightarrow$ is used in type theory, and also to distinguish the internal homs from functor category notation. 
Suppose that $\mathbb{C}$ and $\mathbb{D}$ are $\mathscr{V}$-categories. Recall that the functor category $[\mathbb{C}, \mathbb{D}]$ has objects $S, T: \mathbb{C} \rightarrow \mathbb{D}$ which are $\mathscr{V}$-functors, and that morphism objects are ends

$$
[\mathbb{C}, \mathbb{D}](S, T) \in o b \mathscr{V} \stackrel{\text { def }}{=} \int_{C \in o b \mathbb{C}} \mathbb{D}(S C, T C)
$$

defined using the $\mathscr{V}^{e r}$-hom functor $\mathbb{D}(S(-), T(+)): \mathbb{C}^{o p} \times \mathbb{C} \rightarrow \mathscr{V}^{e r}$. Since we wish to compute concretely with the above end, we recall its definition. For $Y \in o b \mathscr{C}$ there is a subsidiary functor $\mathbb{D}(S(-), T Y): \mathbb{C}^{o p} \rightarrow \mathscr{V}^{e r}$ which is the mate of $M \circ\left(i d \times S_{C, C^{\prime}}\right) \circ \cong$ where $\mathbb{C}^{o p}\left(C^{\prime}, C\right) \times$ $\mathbb{D}\left(S C^{\prime}, T Y\right) \cong \mathbb{D}\left(S C^{\prime}, T Y\right) \times \mathbb{C}^{o p}\left(C^{\prime}, C\right)$ and

$$
\mathbb{D}\left(S C^{\prime}, T Y\right) \times \mathbb{C}^{o p}\left(C^{\prime}, C\right) \stackrel{i d \times S_{C, C^{\prime}}}{\longrightarrow} \mathbb{D}\left(S C^{\prime}, T Y\right) \times \mathbb{D}\left(S C, S C^{\prime}\right) \stackrel{M}{\longrightarrow} \mathbb{D}(S C, T Y)
$$

Taking $Y \stackrel{\text { def }}{=} C^{\prime}$ we define

$$
\sigma_{C, C^{\prime}} \stackrel{\text { def }}{=} \lambda\left(M \circ\left(i d_{\mathbb{D}\left(S C^{\prime}, T C^{\prime}\right)} \times S_{C, C^{\prime}}\right)\right): \mathbb{D}\left(S C^{\prime}, T C^{\prime}\right) \rightarrow \mathbb{C}\left(C, C^{\prime}\right) \Rightarrow \mathbb{D}\left(S C, T C^{\prime}\right)
$$

Similarly we define, by way of $\mathbb{D}(S Y, T(+)): \mathbb{C} \rightarrow \mathscr{V}^{\text {er }}$ with $Y \stackrel{\text { def }}{=} C$,

$$
\rho_{C, C^{\prime}} \stackrel{\text { def }}{=} \lambda\left(M \circ\left(T_{C, C^{\prime}} \times i d_{\mathbb{D}(S C, T C)}\right) \circ \cong^{\prime}\right): \mathbb{D}(S C, T C) \rightarrow \mathbb{C}\left(C, C^{\prime}\right) \Rightarrow \mathbb{D}\left(S C, T C^{\prime}\right)
$$

where $\mathbb{D}(S C, T C) \times \mathbb{C}\left(C, C^{\prime}\right) \cong{ }^{\prime} \mathbb{C}\left(C,{ }^{\prime} C\right) \times \mathbb{D}(S C, T C)$. Thus we have

$$
\Pi_{C \in o b \mathbb{C}} \mathbb{D}(S C, T C) \underset{\sigma}{\stackrel{\rho}{\rightleftarrows}} \Pi_{C, C \in o b \mathbb{C}} \mathbb{C}\left(C, C^{\prime}\right) \Rightarrow \mathbb{D}\left(S C, T C^{\prime}\right)
$$

where $\rho \stackrel{\text { def }}{=}\left\langle\rho_{C, C^{\prime}} \circ p r_{C}\right\rangle$ and $\sigma \stackrel{\text { def }}{=}\left\langle\sigma_{C, C^{\prime}} \circ p r_{C^{\prime}}\right\rangle$ and $\int_{C \in o b \mathbb{C}} \mathbb{D}(S C, T C) \stackrel{\text { def }}{=} E Q R(\rho, \sigma)$.

We shall also write $(-)_{0}: \mathscr{V}-\mathscr{C} \mathscr{A} \mathscr{T} \rightarrow \mathscr{C} \mathscr{A} \mathscr{T}$ for the underlying category functor; recall that $[\mathbb{C}, \mathbb{D}]_{0}=\mathscr{V}-\mathscr{C} \mathscr{A} \mathscr{T}(\mathbb{C}, \mathbb{D})$, the category of $\mathscr{V}$-functors $S, T$ and $\mathscr{V}$-natural transformations $\alpha: S \rightarrow T$ between them.

\section{A.2 Internal category theory}

Please see, for example, Borceux (1994a,b). Let $\mathscr{S}$ be a category with pullbacks (such as $\mathscr{S}$ et, $\mathscr{N}$ om, $\mathscr{F} \mathscr{M} \mathscr{N o m}$ and $\mathscr{F} \mathscr{M} \mathscr{S e t}$ ). Recall that an internal category $\mathbb{A}$ in $\mathscr{S}$ has the following structure

- objects $\mathbb{A}_{0} \in o b \mathscr{S}$ and morphisms $\mathbb{A}_{1} \in o b \mathscr{S}$

- source and target $d_{0}, d_{1}: \mathbb{A}_{1} \rightarrow \mathbb{A}_{0} \in \operatorname{mor} \mathscr{S}$

- identity $\iota: \mathbb{A}_{0} \rightarrow \mathbb{A}_{1} \in$ mor $\mathscr{S}$ and composition $c: \mathbb{A}_{1} \times \mathbb{A}_{0} \mathbb{A}_{1} \rightarrow \mathbb{A}_{1} \in$ mor $\mathscr{S}$, where $\left(\mathbb{A}_{1} \times \mathbb{A}_{0}\right.$ $\left.A_{1}, p r, p r^{\prime}\right)$ is the pullback of $d_{0}$ and $d_{1}$

satisfying the following axioms

(1) $d_{0} \circ \iota=i d_{\mathbb{A}_{0}}=d_{1} \circ \iota$

(2) $d_{1} \circ p r=d_{1} \circ c$ and $d_{0} \circ p r^{\prime}=d_{0} \circ c$

(3) $c \circ h_{0}=i d_{\mathbb{A}_{1}}=c \circ h_{1}$, where $h_{0}$ and $h_{1}$ are factorisations through the pullback $\left(\mathbb{A}_{1} \times \mathbb{A}_{0}\right.$ $\left.\mathbb{A}_{1}, p r, p r^{\prime}\right)$ for cones $\left(\mathbb{A}_{1}, i d_{\mathbb{A}_{1}}, \iota \circ d_{0}\right)$ and $\left(\mathbb{A}_{1}, \iota \circ d_{1}, i d_{\mathbb{A}_{1}}\right)$

(4) $c \circ\left(i d_{\mathbb{A}_{1}} \times \mathbb{A}_{0} c\right)=c \circ\left(c \times \times_{\mathbb{A}_{0}} i d_{\mathbb{A}_{1}}\right)$ where

$$
\begin{aligned}
& \left(i d_{\mathbb{A}_{1}} \times \mathbb{A}_{0} c\right): \mathbb{A}_{1} \times \mathbb{A}_{0}\left(\mathbb{A}_{1} \times \mathbb{A}_{0} \mathbb{A}_{1}\right) \rightarrow \mathbb{A}_{1} \times \mathbb{A}_{0} \mathbb{A}_{1} \\
& \left.\left(c \times \mathbb{A}_{0} i d_{\mathbb{A}_{1}}\right):\left(\mathbb{A}_{1} \times \mathbb{A}_{0} \mathbb{A}_{1}\right) \times \mathbb{A}_{0} \mathbb{A}_{1}\right) \rightarrow \mathbb{A}_{1} \times \mathbb{A}_{0} \mathbb{A}_{1}
\end{aligned}
$$

are factorisations through the pullbacks. 
Given two internal categories $\mathbb{A}$ and $\mathbb{B}$, an internal functor $F: \mathbb{A} \rightarrow \mathbb{B}$ consists of two morphisms $F_{0}: \mathbb{A}_{0} \rightarrow \mathbb{B}_{0}$ and $F_{1}: \mathbb{A}_{1} \rightarrow \mathbb{B}_{1}$ in $\mathscr{S}$ which satisfy the following axioms

(1) $d_{0} \circ F_{1}=F_{0} \circ d_{0}, d_{1} \circ F_{1}=F_{0} \circ d_{1}$

(2) $F_{1} \circ \iota=\iota \circ F_{0}$

(3) $F_{1} \circ c=c \circ\left(F_{1} \times F_{0} F_{1}\right)$, where $\left(F_{1} \times F_{0} F_{1}\right): \mathbb{A}_{1} \times \mathbb{A}_{0} \mathbb{A}_{1} \rightarrow \mathbb{B}_{1} \times \mathbb{B}_{0} \mathbb{B}_{1}$ is the unique mediating morphism such that $p r \circ\left(F_{1} \times_{F_{0}} F_{1}\right)=F_{1} \circ p r$ and $p r^{\prime} \circ\left(F_{1} \times_{F_{0}} F_{1}\right)=F_{1} \circ p r^{\prime}$

Given $\mathbb{A}$ an internal category in $\mathscr{S}$, an internal $\mathscr{S}$-valued functor $\mathrm{P}: \mathbb{A} \rightarrow \mathscr{S}$ is a tuple $\left(P, p_{0}, p_{1}\right)$ where

- $P \in$ ob $\mathscr{S}$ and $p_{0}: P \rightarrow A_{0} \in \operatorname{mor} \mathscr{S}$

- $p_{1}: A_{1} \times_{A_{0}} P \rightarrow P \in$ mor $\mathscr{S}$, where $\left(A_{1} \times A_{0} P, p r_{A_{1}}, p r_{P}\right)$ is the pullback of $d_{0}, p_{0}$

such that the following axioms are satisfied:

(1) $p_{0} \circ p_{1}=d_{1} \circ p r_{A_{1}}$

(2) $p_{1} \circ\left(\iota \circ p_{0}, i d_{P}\right)=i d_{P}$

(3) $p_{1} \circ\left(i d_{A_{1}} \times_{A_{0}} p_{1}\right)=p_{1} \circ\left(c \times_{A_{0}} i d_{P}\right)$

Let $\mathbb{A}$ an internal category in $\mathscr{S}$ and $\mathrm{P}, \mathrm{Q}: \mathbb{A} \rightarrow \mathscr{S}$ two internal $\mathscr{S}$-valued functors specified by $\left(P, p_{0}, p_{1}\right)$ and $\left(Q, q_{0}, q_{1}\right)$ respectively. An internal natural transformation $\alpha: \mathrm{P} \Rightarrow \mathrm{Q}$ is a morphism $\alpha: P \rightarrow Q$ in $\mathscr{S}$ such that the following axioms hold:

(1) $q_{0} \circ \alpha=p_{0}$

(2) $\alpha \circ p_{1}=q_{1} \circ\left(i d_{A_{1}} \times_{A_{0}} \alpha\right)$

Cite this article: Crole RL (2020). The nominal/FM Yoneda Lemma. Mathematical Structures in Computer Science 30, 1011-1024. https://doi.org/10.1017/S0960129520000328 